\title{
Conducción automática de un horno de clínker mediante ordenador de procesos
}

\author{
Dr. DIETER KUHR, SIEMENS, AC. (Erlangen) \\ jUAN R. SANTOdominco, Ledo. en Ciencias Físicas, SIEMEnS, S. A. (Madrid)
}

\section{N T R O D U C C I O N}

En lo que sigue se describe, de forma sucinta, el concepto de conducción automática de un horno Lepol, así como las variaciones introducidas en el mismo para ajustarlo a las características especiales de la instalación, aprovechando las facilidades que para ello ofrece el sistema ADC, de control digital adaptativo. La aplicación descrita se refiere al primer horno de clínker en España, conducido por un ordenador de procesos.

\section{CONFIGURACION DEL EQUIPO (HARDWARE)}

\subsection{Sala del ordenador}

En esta sala se encuentran los siguientes equipos:

- 1 Unidad central, con memoria interna de $16 \mathrm{~K}$-palabras (24 bits).

- 1 Memoria tambor, con una capacidad total de $512 \mathrm{~K}$-palabras (24 bits).

- 1 Teleimpresor de entrada/salida para el diálogo y servicio con el ordenador.

- 1 Elemento combinado de entrada/salida por cinta perforada.

- 1 Impresora rápida para la salida de los protocolos del sistema de información.

- 1 Elemento de proceso con 192 entradas analógicas, 192 entradas digitales y alarmas y 192 salidas digitales.

\subsection{Sala de control}

- 1 Teleimpresor para la entrada/salida de datos, parámetros de regulación etc....

- 1 Teleimpresor de salida para la entrega de información.

En sala aparte se encuentra el espectrómetro multicanal MRS-2 para el análisis, conectado on-line con el ordenador de procesos.

\section{TAREAS ENCOMENDADAS AL ORDENADOR}

Las tareas encomendadas actualmente al ordenador son las siguientes:

- Dosificación del crudo con ayuda de los análisis realizados por el espectrómetro (2 molinos con 4 componentes cada uno).

- Regulación y optimización del grado de llenado de 1 molino de crudo y de 2 molinos de cemento.

- Conducción de un horno Lepol de 1.500 t/día.

- Salida de protocolos y partes de producción. 
La programación ha sido realizada en lenguaje Assembler y en el lenguaje orientado a módulos ADC (control digital adaptativo).

En grandes líneas existen los siguientes sistemas de programas:

- Programas de organización del ordenador.

- Programas para la elaboración de valores de medida.

- Programas de acoplamiento con el espectrómetro y dosificación de crudo.

- Programas para la regulación ADC.

- Programas del sistema de información.

De todos ellos interesa mencionar los siguientes:

\subsection{Elaboración valores de medida}

Para ello se dispone del sistema de programas de estructura modular MADAM el cual, entre otras tareas, hace posible:

- La elaboración normalizada de valores analógicos y binarios.

- La elaboración especial de valores mediante integración, formación de valores medios, control de límites, etc....

De acuerdo con su comportamiento dinámico los valores se leen y elaboran en ciclos de 5,10 y 30 segundos.

\subsection{Sistema ADC}

Mediante este sistema pueden definirse por el teleimpresor de servicio, o por el que está situado en la sala de control, algoritmos de regulación de forma sencilla y rápida.

El sistema dispone aproximadamente de unos 25 módulos para las operaciones básicas de técnica de regulación, los cuales pueden ser ensamblados en la sucesión que se desee. Estos módulos permiten resolver ecuaciones de regulación, operaciones de cálculo y combinaciones lógicas complejas.

La visión completa de la secuencia de regulación se obtiene mediante planos funcionales elaborados según la técnica de figuras adhesivas. En los planos se disponen los módulos ADC en la forma sucesiva que requiere el desarrollo de la regulación. A continuación hay que suministrar a cada módulo sus parámetros individuales.

La construcción de un regulador DDC en el ordenador se realiza mediante las siguientes indicaciones.

- Sucesión de los módulos por aplicar.

- Nombre del regulador.

- Parámetros especiales del regulador.

La conexión del regulador se lleva a cabo mediante una sencilla instrucción de servicio. Durante la marcha se puede entonces (on-line), por variación de cualesquiera de los parámetros realizar el ajuste al proceso. Si el algoritmo de regulación previo no es el adecuado, puede ser borrado y construirse entonces un nuevo regulador con otra estructura.

Disponiendo de la configuración completa del sistema de programas ADC, existe la posibilidad de obtener una identificación automática de tramos de regulación y una optimización de parámetros de los reguladores. 
Está claro que este sistema de programas permite aplicar cualquier concepto de conducción del horno o variar el mismo, por secciones, en un momento determinado. Y esto es lo que se ha hecho en el caso que nos ocupa: partiendo de un concepto básico, éste se ha ajustado a las características específicas del horno.

\subsection{Sistema de información}

Se da salida a partes de producción, conducción y de estado de la instalación. Estos protocolos se emiten, por petición, en cualquier instante y automáticamente todos los días a las 6 de la mañana.

En detalle se tienen los siguientes protocolos:

- Partes diarios de conducción y producción de la sección machaqueo secundario formación "prehomo" crudo.

- Partes diarios de conducción y producción de la sección de la molienda de crudo.

- Partes diarios de conducción y producción de la sección horno Lepol.

- Partes diarios de conducción y producción de la sección de la molienda de cemento.

\section{CONCEPTO DE CONDUCCION DEL HORNO}

Con la implantación del ordenador en las tareas de conducción del horno y mediante la aplicación de algoritmos de regulación ADC se intentaba conseguir:

- una marcha estable del proceso;

- una descarga en el trabajo del personal de sala.

Para ello el ordenador actúa sobre los órganos de ajuste de las siguientes magnitudes:

- Cantidad de gases de salida.

- Alimentación por los platos de granulación.

- Velocidad de la parrilla Lepol.

- Presión diferencial entre cámaras.

- Cantidad de combustible.

- $\mathrm{N} .^{\circ}$ de revoluciones del horno.

- $\quad{ }^{\circ}{ }^{\circ}$ de empujes de las parrillas del enfriador.

- Soplantes del enfriador.

Los ciclos elegidos para cada bucle de regulación DDC oscilaban, según sus características, de 10 a 60 segundos.

\subsection{Regulación de la cantidad de gases de salida}

Con este complejo de regulación se actúa sobre la válvula de mariposa para regular la cantidad de gases que pasan a través del horno y de la parrilla Lepol. El complejo prevé secciones para la vigilancia de la desviación de la regulación, para la vigilancia de la posición de la clapeta y para el cálculo del valor consigna en función de dicha posición.

La cantidad de gases se mide en $\mathrm{m}^{3} / \mathrm{h}$ por un Venturi, haciéndose la corrección por presión y temperatura para obtener los $\mathrm{m}^{3}$ normales. 
En el caso de esta fábrica y, siguiendo los criterios del cliente, la consigna de la clapeta es la de apertura máxima.

\subsection{Regulación de la alimentación}

Con este complejo de regulación se ajusta la cantidad de crudo necesaria, regulándola a partir de la cantidad de gases de salida (en $\mathrm{m}^{3}$ normales) y de la temperatura de gases en la cámara de descarbonatación. Estas indicaciones, junto con el conocimiento del calor específico de los gases, dan la energía calorífica disponible en la parrilla Lepol para el calentamiento y. descarbonatación de los gránulos.

Teniendo en cuenta la posición de la clapeta del ventilador de humos se actúa sobre la alimentación. Si la clapeta está durante mucho tiempo excesivamente cerrada se aumenta la alimentación, disminuyéndose la misma si dicha clapeta está excesivamente abierta durante mucho tiempo.

La instalación dispone de dos platos de granulación con regulación analógica agua-crudo. Como el ventilador se lleva a tope y se considera que la temperatura de recirculación es representativa del aporte calorífico, se toma dicha temperatura como magnitud conductora.

Si el aumento o disminución de la alimentación supera un valor determinado, el incremento positivo o negativo, respectivamente, se reparte a partes iguales entre ambos platos. Si quedan por debajo de dicho valor, los pequeños ajustes resultantes se efectúan siempre sobre el plato que tiene menos material, en el caso de ser incrementos positivos, y sobre el de más material, cuando sean incrementos negativos.

\subsection{Regulación de la parrilla Lepol}

Los algoritmos previstos para el precalentador Lepol actúan sobre la velocidad de la parrilla y sobre el ventilador del aire de recirculación.

El primero de estos algoritmos regula, mediante el ajuste de la velocidad de la parrilla, la altura de la tolva de entrada del material. En el segundo, y mediante la clapeta de entrada de aire de recirculación, se ajusta la presión diferencial entre cámaras.

Existe un regulador analógico en back-up.

\subsection{Regulación del enfriador}

Se distinguen dos grupos principales:

\subsubsection{Regulación de la velocidad de las parrillas}

El enfriador tiene dos parrillas. Con esta regulación se actúa sobre la velocidad de las parrillas, en función de la presión en la primera cámara de cada parrilla, para conseguir que los lechos de clínker en ellas sean constantes, consiguiendo con ello un enfriamiento uniforme y, en el caso de la primera parrilla, se influye sobre la temperatura del aire secundario.

En las primeras secciones de este algoritmo se realiza una vigilancia del total y del gradiente de las presiones.

Dependiendo de estas magnitudes se efectúa una elección entre dos tramos de regulación diferentes. 


\subsubsection{Regulación de las soplantes del enfriador}

Con este complejo de regulación se condicionan el aire secundario y el enfriador. En las primeras secciones se mantiene en un calor fijo la cantidad de aire frío en el primer ventilador. Esta indicación, junto con la del combustible utilizado, sirven para calcular el valor consigna para la regulación del caudal de aire frío del ventilador 2. En dichas consignas de caudales se hacen correcciones en dependencia con la temperatura de las placas de la parrilla. El caudal del tercer ventilador se mantiene constante. Las consignas de caudales de los ventiladores 4 y 5 se calculan en base a la temperatura de salida del enfriador, lo que se puede considerar como una regulación de la temperatura del clínker.

Para la presión en caperuza se dispone de una regulación analógica.

\subsection{Regulación del combustible}

En este algoritmo se distinguen dos formas de regulación, según se encuentre el horno en condiciones normales o aparezcan caídas de temperatura en zona. Esta sección de regulación tiene que garantizar una temperatura en zona de sinterización necesaria para obtener una calidad de clínker preestablecida.

En la conducción normal la regulación del combustible se realiza en función de la temperatura en zona con corrección por falta de $\mathrm{O}_{2}$ y con una actuación enérgica en el caso de que aparezca $\mathrm{CO}$.

Existen dos tramos distintos de regulación según sea la tendencia de la temperatura. Si ésta aumenta demasiado la disminución de la cantidad de combustible se realiza a pequeños pasos y, por el contrario, si baja la temperatura en zona se aumenta rápidamente la cantidad de combustible.

Para detectar los casos de caída de temperatura en zona se le fijan a ésta tres valores límites a partir de la temperatura consigna, que indican tres caídas de la misma de distinta intensidad.

Según sea el límite desbordado inferiormente se toman medidas correctas de distinta intensidad actuando sobre los ajustes de:

- la cantidad de fuel;

- la cantidad de fuel y la velocidad del horno;

- la cantidad de fuel, la velocidad del horno y la alimentación.

En el caso específico de esta instalación se actúa únicamente sobre la cantidad de fuel, porque la comprobación de la actuación sobre la velocidad sólo se podrỉa hacer en casos muy esporádicos; y sobre la alimentación, tal como se dijo anteriormente, no se actúa por temperatura en zona. 
1. Se inició bajo la presidencia del Sr. MESSA y lo abrió el Sr. COROMINA, quien planteó dos cuestiones. Una, sobre cómo se tiene que proceder en casos en que sea necesario reparar el ordenador, ya sea por causas propias del mismo, o por averías ajenas a él, como por ejemplo paradas de la fábrica.

Una vez aclarado que la pregunta se refería al "back-up" de la fábrica, contestó el Sr. SANTODOMINGO que el sistema de relación que se utiliza es tal que, si por ejemplo se paran los molinos, o fallan instrumentos de alguna sección, permite que secciones parciales de la zona afectada del proceso se puedan llevar manualmente, o regularse en vacantes del panel desde la sala de control, de forma que el ordenador sigue mandando el resto no afectado. Yo - dijo-, he mencionado una serie de dispositivos que tiene la fábrica en "back-up", en el caso de la parrilla LEPOL y de los platos de granulación. Naturalmente, si se avería el ordenador, todo él se desconecta; pero en el caso de que falle el horno, por ejemplo, el ordenador sigue trabajando con la dosificación -si hay más hornos que alimentar-, y con el grado de llenado de los molinos; o viceversa. No se -añadió-, si esto contesta a la pregunta, porque yo no soy de fábrica y no sé en realidad qué es lo que tiene en "back-up".

La segunda cuestión planteada por el Sr. COROMINA se refirió al cálculo de la dosificación del crudo, preguntando si, además de los análisis y del control del tonelaje, hay algún otro parámetro interesante que intervenga en dicho cálculo.

El Sr. SANTODOMINGO remitió la pregunta al Sr. MAGALLON, quien respondió que la dosificación se regula en función del análisis de la muestra tomada en el MRS y, naturalmente, en función de una serie de valores de consigna que se establecen (por ejemplo, de los módulos que se quieren conseguir), y que los ajustes finales se hacían también en función de valores reales de dosificación con que en cada momento dado actúan las básculas. Es decir - puntualizó-, se analiza la muestra tomada, se calculan las correcciones que hay que hacer en los tantos por ciento de la dosificación, y se envían a las básculas los correspondientes tantos por ciento corregidos; por lo tanto - dijo-, los tres tipos de parámetros o magnitudes que intervienen son: los análisis de las muestras, los valores de consigna que se imponen, y los valores reales de dosificación con que actúan las básculas en los momentos a los que corresponden los análisis de las muestras.

El Sr. COROMINA preguntó de nuevo si, desde el punto de vista del análisis, los valores de consigna se refieren a dos o a los tres módulos, a lo que el Sr. MAGALLON contestó que a dos módulos y a la relación de un tercero (ya que el tercer módulo o módulo de fundentes está ligado con el de silicatos, por haberse establecido una dependencia entre ambos), y al grado de saturación.

2. El Sr. BOSQUET, en relación con la temperatura de la zona de cocción, como variable en la marcha del horno, preguntó mediante qué sistema o aparatos se determina, y si su determinación es fiable.

Le respondió al Sr. SANTODOMINGO diciendo que él consideraba que el aparato "ARDOCOL" lo era, pero que sería mejor preguntárselo a los técnicos de fábrica presentes en la sala. 
Concretó más su pregunta el Sr. BOSQUET, refiriéndola a hornos de vía seca y grardes producciones, erı los que hay una atmósfera con mucho polvo, y recabó opiniones acerca de si el conocido aparato "ARDOCOL" proporciona valores fiables hasta el punto de poder basar en ellos la marcha del horno. Insistió el Sr. SANTODOMINGO en su respuesta y trasladó la cuestión al Sr. KUHR. Este dijo que cuando no hay mucho polvo en el horno la medida de la temperatura es fiable, pero que cuando hay mucho polvo ya no lo es tanto; en tal caso hay que hacer un estudio basado en una serie de medidas para establecer una relación que permita, en función de la influencia del polvo, efectuar unas correcciones en el sistema, a fin de tener un valor representativo de la temperatura.

Amplió aún más su pregunta el Sr. BOSQUET inquiriendo cuántos aparatos del tipo del citado existen instalados en todo el mundo y funcionando satisfactoriamente. La respuesta conjunta de los Sres. KUHR y SANTODOMINGO fue que las fábricas automatizadas con equipos SIEMENS, aun siendo de distintos suministradores, van provistas de aparatos "ARDOCOL" y que se ha suministrado un centenar de ellos.

Nueva insistencia del Sr. BOSQUET sobre si funcionan con resultados satisfactorios, o si en la mayoría de los casos hay que recurrir a algún otro procedimiento. Y nueva respuesta del Sr. KUHR en el sentido de que el aparato en sí funciona muy bien. Y que dadas las características de cada horno, al instalar el aparato hay que hacer pruebas y ajustes para comprobar si la temperatura que el aparato marca es representativa o no, a causa del polvo en el horno o de cualquier otro problema que pueda surgir. Es decir -añadió-, hay que llevar a cabo un estudio de los valores de medida que el aparato va dando y, a partir del mismo, hallar una relación de forma que en base a tal magnitud se pueda conducir el horno. Si se tiene instalado un ordenador de procesos - continuó-, se puede regular muy bien la temperatura, haciendo mediciones de ella en cada instante y estudiando estas mediciones para comprobar si la temperatura es representativa. Si lo es, se funciona con ella durante un largo período y, cuando deja de serlo por haberse efectuado un cambio de material o por cualquier otra causa, entonces hay que poner otro valor que sustituya el anterior, es decir, hay que variar también el correspondiente valor de consigna.

Finalmente, el Sr. BOSQUET se interesó por la vida media y por los cuidados de mantenimiento que requieren los aparatos, siéndole respondido, en cuanto al primer punto, que su promedio era de cinco años; y en cuanto al segundo, que la electrónica ha de estar refrigerada constantemente y que la óptica ha de mantenerse limpia de polvo, mediante aire a presión.

3. El Sr. DANEYKO, respecto de la regulación del horno y de la conducción automática de la planta, preguntó después si se emplea instrumentación de medida más o menos convencional, o si se utilizaba algo especialmente nuevo, respondiéndole los Sres. KUHR y SANTODOMINGO que la técnica de medida empleada era la convencional, a base de analizadores de gases, medidores de temperatura, etc., y que existía un panel o cuadro de control muy enriquecido. A la nueva pregunta del Sr. DANEYKO acerca del nivel a que se produce la separación de las señales que van al ordenador y las que van al cuadro, respondieron que son las mismas señales las que van a un sitio y otro.

Se añadió después — por parte del Sr. MAGALLON - que la fábrica a la que se refiere la instalación de conducción por ordenador que habían descrito los ponentes, no es una fábrica de nuevo diseño para la que desde el principio se ha proyectado y acoplado el ordenador, sino que se trata de una fábrica que estaba en servicio y poseía ya una instrumentación, una sala de control centralizado y un ordenador que desde hacía años llevaba la dosificación. Al introducir después la conducción del horno y la regulación de los molinos se aprovechó toda la instrumentación existente, y sólo en casos muy especia- 
les se completó al no existir algún determinado punto de medida necesario, o se revisó la fiabilidad del ya existente, a fin de meterlo en el ordenador.

4. A continuación preguntó el Sr. GASCON acerca del aumento de producción que pudo suponer el paso de la instalación sin ordenador a la instalación con él. Respondió el Sr. SANTODOMINGO que la respuesta no le correspondía, y que la regulación y óptimación del grado de llenado de los molinos y la marcha del horno estaban en servicio en la mencionada fábrica desde hacía unos meses, y que se estaba procediendo a una toma de datos y a una evaluación de resultados a lo largo de unos siete meses - para que unos y otros fueran representativos-; que todo ello se daría a conocer más adelante a través de una publicación.

5. Después el Sr. PUIG dijo que se había descrito la conducción automática del horno "en condiciones normales", mediante el ordenador y los parámetros correspondientes: cantidad de gases, velocidad de parrilla, presión diferencial, cantidad de combustible, revoluciones del horno, etc. Preguntó cuál era la forma de actuar cuando las condiciones no eran "normales".

El Sr. SANTODOMINGO quiso aclarar si el Sr. PUIG se refería a casos de caída de temperatura en la zona de clinkerización o a la marcha del horno en general, siendo puntualizado por el Sr. PUIG que se refería a cualquier tipo de alteración que se pudiera producir, dando lugar a una marcha no regular, o uniforme, o "normal" del horno. El Sr. SANTODOMINGO dijo que, si no hay problemas mecánicos en los platos de granulación ni se calientan demasiado las placas de la parrilla - que para evitarlo hay dispositivos de vigilancia-, y si el ordenador regula bien la alimentación, eso se puede considerar como una conducción normal. Pero que los problemas aparecen siempre cuando se producen caídas de temperatura en la zona de clinkerización, para cuyo caso se fijan límites, tanto en la caída como en la recuperación de la temperatura, dados por unas curvas. Y que para mantenerse dentro de esos límites se actúa primero sobre el combustible, aumentando la alimentación del mismo; si a pesar de ello la temperatura sigue cayendo en la zona de clínkerización, se actúa entonces sobre el número de revoluciones del horno, reduciéndolas, y por último, en el peor de los casos, se actúa sobre la alimentación reduciéndola también. Todo esto —dijo—, se refleja después en la subida y recuperación de la temperatura, a partir de la cual se vuelve poco a poco a actuar en sentido inverso, esto es, restableciendo la alimentación primitiva, aumentando la velocidad del horno y reduciendo la cantidad de combustible. En la fase de puesta en marcha - concluyó el Sr. SANTODOMINGO-, en que todavía no está hecho el necesario ajuste de parámetros, se hace preciso a veces desconectar el ordenador, teniendo que actuar el personal de la sala de control.

El Sr. PUIG insistió en preguntar cómo se podría conducir el horno en condiciones que no fuesen las normales, por ejemplo, cuando la temperatura medida en la zona de clinkerización no sea fiable a causa del polvo en la atmósfera del horno, si se trata de uno con recuperación de calor a base de ciclones. Los ponentes contestaron que, dejando aparte la fiabilidad del aparato de medida de temperatura que se utilice, la cuestión planteada se puede resolver a base de toma de datos y de la evaluación de los mismos de que antes se había hablado, y que en el caso de un horno con intercambiadores se aplicaban los mismos conceptos, añadiendo que así llevaba dos años y medio de funcionamiento sin perturbaciones la fábrica de la Heidelberg Zement.

El Sr. PUIG hizo finalmente una pregunta relacionada con el control de calidad. Si éste se basa - dijo-, en el análisis de las muestras de crudo por fluorescencia de rayos X ¿hay medio de poder continuar haciéndolo, por ejemplo pasando al análisis tradicional de carbonatos en el caso de que el equipo de rayos $\mathrm{X}$ se averíe? ¿Se llevan en paralelo 
ambos tipos de análisis para pasar del de fluorescencia al tradicional en caso necesario y sin solución de continuidad?

Trasladada la pregunta por el Sr. SANTODOMINGO al Sr. MAGALLON, éste dijo que el análisis de carbonatos se abandonó hace años, al entrar en servicio los ordenadores, y que cuando el espectrógrafo se avería no sabía realmente si se procedía a efectuar análisis químicos periódicos o a determinar carbonatos, ya que el tema correspondía a la explotación del proceso en fábrica y que le era ajeno.

Finalmente, el Sr. SANTODOMINGO transmitió una aclaración del Sr. KUHR en relación con la mencionada fábrica de la Heidelberg Zement, en la que han conseguido conducir un horno con intercambiadores durante varios meses sin necesidad de recurrir a ninguna operación manual, aplicando el criterio de conducción para el caso de caída de temperatura en la zona de clinkerización. Y que de ello se había hecho un estudio publicado en la revista alemana Zement-Kalk-Gips en 1975.

Y no habiendo más preguntas que responder, el Presidente, Sr. MESSA, agradeció el interés de los participantes en el Coloquio y dió este por concluido. 